\title{
Dynamics Modeling of Spacial Flexible Manipulator Basing on Absolute Nodal Coordinate Formation
}

\author{
MA Chao ${ }^{1, *}$, ZHANG Yue $^{1}$, WEI Cheng ${ }^{1}$, ZHAO Yang $^{1}$ \\ ${ }^{1}$ Dept. of Aerospace Engineering, Harbin Institute of Technology, Harbin, 150001, China
}

Keywords: Manipulator; Flexible; Absolute coordinate; Dynamics

\begin{abstract}
Adopting absolute nodal coordinate method, this article establishes dynamic model of double-connecting rod flexible manipulator. Its broad sense moment is obtained through virtual work principle. State equation is further deducted on this basis and numerical integration method is given. This article adopts PID control strategy to realize circular trace at the end of manipulator and square tracing control. It is discovered from the comparison of absolute nodal coordinate method with traditional hypothesis model that the absolute nodal coordinate method can get a more accurate result.
\end{abstract}

\section{Introduction}

For the current orbital net dynamics, the initial rope net dynamics and collision dynamics models have been finished and ground casting net experiment and corresponding method study on capture strategies and performances analysis have been implemented. But because of adopting dynamics models such as simplified finite segment or particle spring, there is no accurate representation of the nature of constitutive model of rope net model and there is no implementation of ground experiment to correct or verify constitutive model of rope net, so it is difficult to ensure the following performance analysis as well as the correctness and efficiency of casting net strategy, and there is a lack of evidences that dynamics models plays a guidance role to casting net experiment and casting experiment has can correct dynamics model. In order to solve the correctness of this model and make up the difference between this model and experiment, it is very important to implement research and verification of dynamics model for orbital net dynamics mode which is also the foundation of all the successive analyses.

\section{Dynamics Modeling}

\section{Movement of soft cable element}

Establish soft cable element following the typical Euler-Bernoulli Beam Theory Hypothesis in which the cross section of beam is rigid plane and always vertical to the axis of the beam. In Euler-Bernoulli Beam Theory, the axial deformation, bending deformation and torsional deformation should all be considered. The cutting deformation can be neglected. Because there are tiny influences of torsional deformation of winding soft cable axis on the whole dynamics, on the basis of Euler-Bernoulli Beam Theory Hypothesis, the simpler soft cable element can be obtained if only considering axial deformation and bending deformation by neglecting torsional deformation. At this moment the soft cable axis can completely be used to describe the movement of soft cable. 


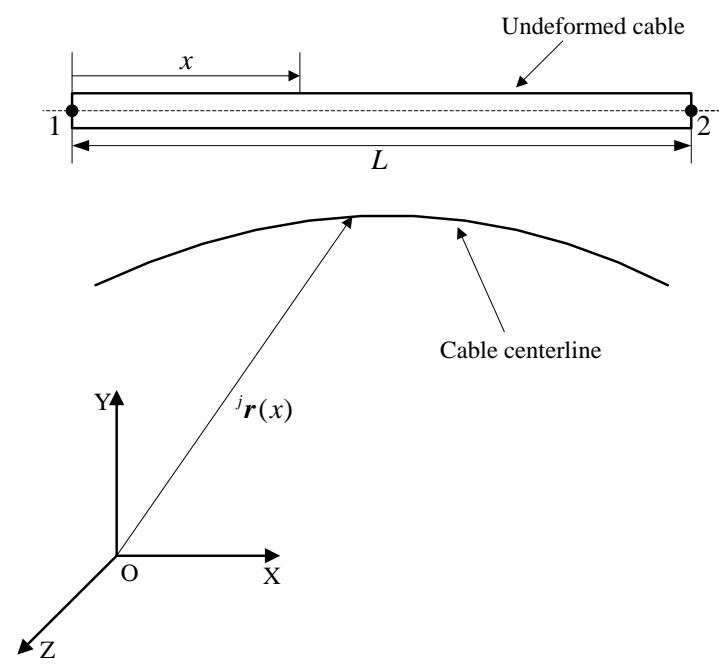

Fig.1. Soft cable element model

At this moment, basing on continuum mechanics method, the position of an arbitrary point on centerline of soft cable can be expressed as:

$$
{ }^{j} \boldsymbol{r}=\left[\begin{array}{c}
{ }^{j} r_{1} \\
{ }^{j} r_{2} \\
{ }^{j} r_{3}
\end{array}\right]=\left[\begin{array}{c}
a_{0}+a_{1} x+a_{2} x^{2}+a_{3} x^{3} \\
b_{0}+b_{1} x+b_{2} x^{2}+b_{3} x^{3} \\
c_{0}+c_{1} x+c_{2} x^{2}+c_{3} x^{3}
\end{array}\right]
$$

The superscript $\mathrm{j}$ means No.j soft cable element.

It is known from the equation that position vector ${ }^{j} \boldsymbol{r}$ is a function related to parameter $X, X$ is material coordinate of soft cable under undeformed status.

The original ANCF soft cable element should consider axial deformation, bending deformation, cutting deformation and torsional deformation. Each element has 24 degrees of freedom with each end having 12. The ANCF soft cable element of this time only considers axial deformation and bending deformation. The broad sense coordinate of soft cable element uses the position and material derivative of the two ends of the element. The degrees of freedom of the element is a half of the original ANCF which enhances computation efficiency without influencing computation accuracy. Let the length of the element be $L$, the broad sense coordinate of soft cable element is :

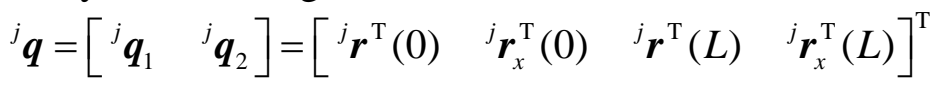

${ }^{j} \boldsymbol{r}(0)$ and ${ }^{j} \boldsymbol{r}_{x}(0)$ indicate position vector and gradient vector of 1 at the end respectively. And ${ }^{j} \boldsymbol{r}(L)$ and ${ }^{j} \boldsymbol{r}_{x}(L)$ indicate position vector and gradient vector of 2 at the end respectively.

The position vector of a point $(x)$ on the centerline of soft cable element can be expressed with broad sense coordinate as:

${ }^{j} \boldsymbol{r}(x, t)=\boldsymbol{S}(x){ }^{j} \boldsymbol{q}(t)$

The $\boldsymbol{S}(x)$ is shape function of three-dimensional soft ANCF soft cable element whose specific form is as follows:

$$
\begin{aligned}
& \boldsymbol{S}(x)=\left[\begin{array}{cccccc}
1-3 \xi^{2}+2 \xi^{3} & 0 & 0 & L\left(\xi-2 \xi^{2}+\xi^{3}\right) & 0 & 0 \\
0 & 1-3 \xi^{2}+2 \xi^{3} & 0 & 0 & L\left(\xi-2 \xi^{2}+\xi^{3}\right) & 0 \\
0 & 0 & 1-3 \xi^{2}+2 \xi^{3} & 0 & 0 & L\left(\xi-2 \xi^{2}+\xi^{3}\right)
\end{array}\right. \\
& \left.\begin{array}{cccccc}
3 \xi^{2}-2 \xi^{3} & 0 & 0 & L\left(-\xi^{2}+\xi^{3}\right) & 0 & 0 \\
0 & 3 \xi^{2}-2 \xi^{3} & 0 & 0 & L\left(-\xi^{2}+\xi^{3}\right) & 0 \\
0 & 0 & 3 \xi^{2}-2 \xi^{3} & 0 & 0 & L\left(-\xi^{2}+\xi^{3}\right)
\end{array}\right]
\end{aligned}
$$

Among it $\xi=\frac{x}{L}$. 


\section{Element functions}

Because the shape function of soft cable element is constant, the speed vector of an arbitrary point on the soft cable can be written as

$$
{ }^{j} \dot{\boldsymbol{r}}=\boldsymbol{S}^{j} \dot{\boldsymbol{q}}
$$

Using equation, the kinetic energy of soft cable element can be written as:

$$
\begin{aligned}
{ }^{j} T & =\frac{1}{2} \int_{0}^{L} \rho \int_{A}{ }^{j} \dot{\boldsymbol{r}}^{\mathrm{T}}{ }^{j} \dot{\boldsymbol{r}} d A d x=\frac{1}{2} \int_{0}^{L} \rho \int_{A}{ }^{j} \dot{\boldsymbol{q}}^{\mathrm{T}} \boldsymbol{S}^{\mathrm{T}} \boldsymbol{S}^{j} \dot{\boldsymbol{q}} d A d x=\frac{1}{2}{ }^{j} \dot{\boldsymbol{q}}^{\mathrm{T}}\left(\int_{0}^{L} \rho\left(A \boldsymbol{S}^{\mathrm{T}} \boldsymbol{S}\right) d x\right) \square^{j} \dot{\boldsymbol{q}} \\
& =\frac{1}{2}{ }^{j} \dot{\boldsymbol{q}}^{\mathrm{T}}{ }^{j} \boldsymbol{M}^{j} \dot{\boldsymbol{q}}
\end{aligned}
$$

The $\rho$ and A is the density and cross sectional area respectively of soft cable element. ${ }^{j} \boldsymbol{M}=\int_{0}^{L} \rho\left(A \boldsymbol{S}^{\mathrm{T}} \boldsymbol{S}\right) d x$ is constant mass matrix of ANCF soft cable element which are expressed specifically as follows:

$$
{ }^{j} \boldsymbol{M}=\int_{0}^{L} \rho\left(A \boldsymbol{S}^{\mathrm{T}} \boldsymbol{S}\right) d x=m\left[\begin{array}{cccc}
\frac{13}{35} \mathbf{I} & \frac{11}{210} L \mathbf{I} & \frac{9}{70} \mathbf{I} & -\frac{13}{420} L \mathbf{I} \\
& \frac{1}{105} L^{2} \mathbf{I} & \frac{13}{420} L \mathbf{I} & -\frac{1}{140} L^{2} \mathbf{I} \\
& & \frac{13}{35} \mathbf{I} & -\frac{11}{210} L \mathbf{I} \\
\text { sym } & & & \frac{1}{105} L^{2} \mathbf{I}
\end{array}\right]
$$

\section{Internal energy of element}

Using Bernoulli-Euler beam formulation, the elastic energy of soft cable element is:

$$
{ }^{j} U=\frac{1}{2} \int_{0}^{L}\left(E A^{j} \varepsilon_{0}{ }^{2}+E J_{\kappa}{ }^{j} \kappa^{2}\right) d x
$$

The $E$ is elastic modulus, $J_{\kappa}$ is the inertia moment of cross section of soft cable, axial strain ${ }^{j} \varepsilon_{0}$ and curvature ${ }^{j} \kappa$ can be obtained through the following two formulas

$$
\begin{aligned}
& { }^{j} \mathcal{E}_{0}=\sqrt{{ }^{j} \boldsymbol{r}_{x}{ }^{\mathrm{T}} \boldsymbol{r}_{x}}-1 \\
& { }^{j} \boldsymbol{\kappa}=\frac{\left|\boldsymbol{r}_{x} \times{ }^{j} \boldsymbol{r}_{x x}\right|}{\left|{ }^{j} \boldsymbol{r}_{x}\right|^{3}}
\end{aligned}
$$

\section{Experimental and Analysis}

The initial state of simple pendulum beam is shown in Figure 2. The simple pendulum is fixed with revolute pair and can oscillate freely within the plane. The initial position of single pendulum is horizontal. It oscillates freely under the effect of gravity with an initial speed of $0 \mathrm{~m} / \mathrm{s}$. The length of simple pendulum is $1.2 \mathrm{~m}$ with an acceleration of gravity of $9.81 \mathrm{~m} / \mathrm{s} 2$. As an initial study, simulate uniform section beam, first boundary variable section beam, second boundary variable section beam and hollow beam respectively. Analyze the influences of element numbers on the simulation time and simulation accuracy of the system through changing the number of beam element. Analyze influences of section parameters on the performances of system dynamics. 


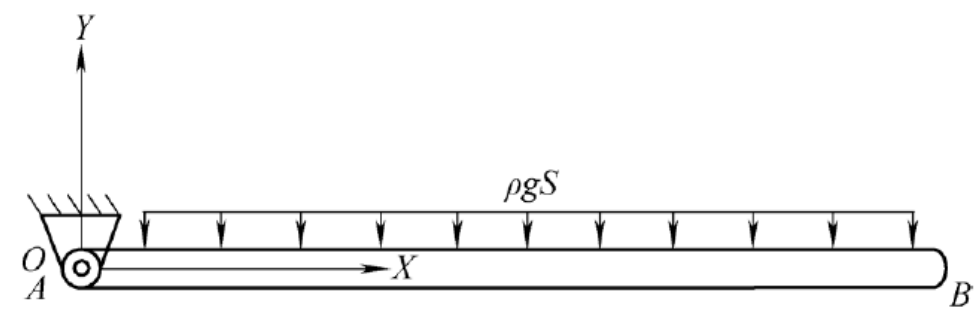

Fig.2. Free oscillation of flexibly simple pendulum

Take first variable section simple pendulum beam as an example whose structural sketch is shown as Figure 5b. The number of detailed parameters is given below. Divide the simple pendulum into 1 element, 5 elements and 10 elements respectively. When dividing into 1 element, the system includes 2 nodes with each including 6 node coordinates. The end A includes a mobile constraint. So there are altogether 10 broad sense coordinates. When dividing into 5 elements, the system includes 10 nodes with each also including 6 node coordinates. The end A include a mobile constraint and every two elements include fixed constraints. So there are altogether 34 node coordinates in the system. Similarly, when dividing into 10 element, the system includes 64 node coordinates. The elastic modulus of the material taken is $0.7 \mathrm{MPa}$, Poisson's ratio is 0.3 and density is $5540 \mathrm{~kg} / \mathrm{m} 3$.

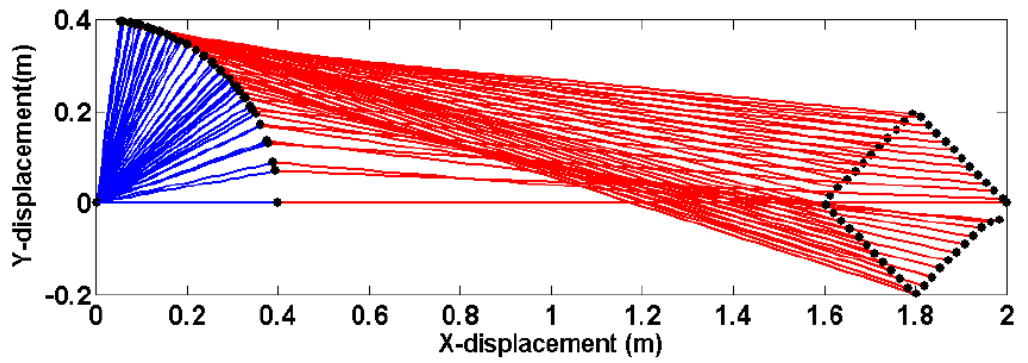

Fig.3. Configuration of manipulator

Because of different numbers chosen, the displacement of flexibly simple pendulum end is not completely identical and has obvious differences with rigid simple pendulum. The displacement diagram of simple pendulum dividing into 5 elements almost coincides with simple pendulum dividing into 10 elements. The simple pendulum dividing into 1 element has certain differences with the above two. In order to compare the differences of deformation of all flexible quantity, compute the maximum deformation in simple pendulum movement process and deformation when the simple pendulum in the lowest position when the element number is different. The length after simple pendulum deformation from end point is similar to the distance to fixed end.

Actually, because the simple pendulum will have bending deformation, so the actual deformation is larger than calculated value. Table 1 indicates the lengths of simple pendulum under various conditions. Comparing with the original length of simple pendulum which is $1.2 \mathrm{~m}$, the maximum deformation of simple pendulum which is divided into 1 element is $11.38 \%$ of the original length. The maximum deformation of simple pendulum which is divided into 5 elements is $12.20 \%$ of the original length. And maximum deformation of simple pendulum which is divided into 10 elements is $12.22 \%$ of the original length. It can be seen the deformation increases with the increase of element number. The deformation of simple pendulum with 1 element simple pendulum is smaller that the other two flexible beams which indicates the rigidity of simple pendulum dividing into 1 element is greater than simple pendulums dividing into 5 elements and 10 elements. The results of simple pendulums dividing into 5 elements and 10 elements are similar. Similarly, the deformation at the lowest place also shows the same rules.

It is indicated from the above results that smaller element number will increase rigidity of system. The increase of element number can increase accuracy of simulation. 


\section{Conclusion}

This article establishes beam element dynamics analysis model basing on absolute node coordinate method. The deformation of beam element is described by deformation gradient form which considers cutting deformation. Also it introduces the characteristics of variable sections of beam element and establishes mass matrix and rigid matrix of variable section of beam element. This article analyzes the influences of element number on the computation efficiency, computation accuracy by using simulation case and comparison of beam elements with different element numbers, as well as analyzes influences of section parameter change on dynamics performances of the system through comparing beam elements of different section forms.

\section{Reference}

[1] Y. Geng, J. Chen, K. Pahlavan, Motion detection using RF signals for the first responder in emergency operations: A PHASER project[C], 2013 IEEE 24nd International Symposium on Personal Indoor and Mobile Radio Communications (PIMRC), London,Britain Sep. 2013

[2] Lv. Zhihan, Liangbing Feng, Haibo Li, and Shengzhong Feng. "Hand-free motion interaction on Google Glass." In SIGGRAPH Asia 2014 Mobile Graphics and Interactive Applications, p. 21. ACM, 2014.

[3] Zhong. Chen, Stefan Müller Arisona, Xianfeng Huang, Michael Batty, and Gerhard Schmitt. "Detecting the dynamics of urban structure through spatial network analysis." International Journal of Geographical Information Science 28, no. 11 (2014): 2178-2199.

[4] Li. Wubin, Johan Tordsson, and Erik Elmroth. "An aspect-oriented approach to consistency-preserving caching and compression of web service response messages." In Web Services (ICWS), 2010 IEEE International Conference on, pp. 526-533. IEEE, 2010. 